\title{
High PARP-1 expression is associated with tumor invasion and poor prognosis in gastric cancer
}

\author{
YING LIU ${ }^{1}$, YU ZHANG ${ }^{1}$, YING ZHAO ${ }^{2}$, DONGNA GAO ${ }^{1}$, JING XING $^{1}$ and HUI LIU ${ }^{1}$ \\ ${ }^{1}$ Emergency Department, The First Affiliated Hospital of Dalian Medical University, Dalian, Liaoning 116011; \\ ${ }^{2}$ Department of General Surgery, Shengjing Hospital of China Medical University, Shenyang, Liaoning 110004, P.R. China
}

Received July 23, 2015; Accepted July 12, 2016

DOI: $10.3892 / 01.2016 .5169$

\begin{abstract}
Poly (adenosine diphosphate-ribose) polymerase 1 (PARP-1) was previously demonstrated to be overexpressed in numerous malignant tumors and associated with invasiveness and poor prognosis. However, the expression of the PARP-1 protein in gastric cancer and its association with clinical outcomes requires further investigation. In the present study, the expression of PARP-1 in 564 gastric cancer tissues and 335 tumor-adjacent control tissues is investigated, using tissue microarray-based immunohistochemistry. PARP-1 expression levels were demonstrated to be significantly higher in gastric cancer tissue samples, as compared with control tissue samples. In gastric cancer, high PARP-1 expression levels were significantly associated with Helicobacter pylori (H. pylori) infection $(\mathrm{P}=0.032)$, decreased differentiation $(\mathrm{P}<0.001)$, increased depth of invasion $(\mathrm{P}=0.037)$, presence of lymphatic invasion $(\mathrm{P}<0.001)$, presence of lymph node metastasis $(\mathrm{P}<0.001)$, and advanced tumor-node-metastasis (TNM) stage $(\mathrm{P}=0.015)$. High PARP-1 expression levels were associated with a significantly shorter overall survival rate $(\mathrm{P}<0.001)$ and disease-free survival rate $(\mathrm{P}=0.001)$ in patients with gastric cancer, particularly a subset of patients with $H$. pylori infection or an advanced TNM stage. In addition, univariate analysis indicated that PARP-1 high expression levels were significantly associated with a poor prognosis in gastric cancer. These results suggest that PARP-1 expression may be involved
\end{abstract}

Correspondence to: Dr Hui Liu, Emergency Department, The First Affiliated Hospital of Dalian Medical University, 222 ZhongShan Road, Dalian, Liaoning 116011, P.R. China

E-mail: liuhui_dmu@163.com

Abbreviations: AUCs, areas under curve; CI, confidence interval; DFS, disease-free survival; EMT, epithelial-mesenchymal transition; H. pylori, Helicobacter pylori; IRS, immunoreactivity score; OS, overall survival; PARP-1, poly (adenosine diphosphate-ribose) polymerase 1; ROC, receiver operating characteristic; RR, relative risk; TNM, tumor-node-metastasis; TMA, tissue microarray

Key words: gastric cancer, poly (adenosine diphosphate-ribose) polymerase $1, H$. pylori infection, metastasis, prognosis in the progression and prognosis of gastric cancer, particularly H. pylori-positive or advanced-stage gastric cancer.

\section{Introduction}

Human cancers constitute a notable burden on societies. In less developed countries, gastric cancer among males is one of the leading causes of cancer-associated mortalities (1). Due to its genetic complexity and heterogeneity, advances in the treatment of gastric cancer have been limited thus far (2). Therefore, the identification of specific biomarkers is crucial for the development of individualized treatments, which are required for the effective and precise management of gastric cancer in patients.

The poly (adenosine diphosphate-ribose) polymerase (PARP) proteins are a family of 17 enzymes involved in the regulation of transcription, DNA damage response, genome stability, cell cycle, energy metabolism, cell death and tumorigenesis (3-5). PARP-1 was the first PARP enzyme identified over 50 years ago and has been the subject of several studies (6-8). PARP-1 binds single- or double-stranded DNA breaks; its activity increases as required to maintain genomic integrity $(9,10)$. It has previously been demonstrated that PARP-1 is overexpressed in numerous types of tumors, including malignant melanomas, colorectal cancer, breast cancer, testicular tumors and lymphangioleiomyomatosis, and that it is associated with invasiveness and poor clinical prognosis (11-15). Therefore, PARP-1 may be a potential anticancer target $(16,17)$. PARP inhibitors are also currently used in combination with chemotherapeutic agents to increase tumor responses (18-20).

PARP-1 single nucleotide polymorphisms, including PARP-1 2819G, PARP-1 762Val/Ala and PARP-1 rs1136410, were previously demonstrated to be associated with gastric cancer susceptibility and lymph node metastasis in gastric cancer (21-23). Le et al (24) demonstrated that PARP-1 inhibitors enhance the cytotoxicity of cisplatin in human gastric cancer cells. Liu et al (25) previously demonstrated that the cochinchina momordica seed extract significantly inhibited the survival rate of human gastric cancer cells by downregulating PARP expression. However, the protein expression pattern of PARP-1 in gastric cancer patients requires further study. It remains to be determined whether the expression levels of PARP-1 are associated with the tumorigenesis and progression of gastric cancer. 
In the present study, tissue microarray-based immunohistochemistry was used to determine the expression of PARP-1 in 564 gastric cancer tissue samples and 335 tumor-adjacent tissue samples. The aim of the current study was to analyze the association between the expression levels of PARP-1 and the clinicopathological features and prognosis of gastric cancer patients.

\section{Materials and methods}

Patients and tissue samples. Human gastric cancer tissue samples were obtained from 564 patients (405 males and 159 females; age range, 29-82 years) with primary gastric tumors who underwent D1 or D2 radical gastrectomy surgery at the First Affiliated Hospital of Dalian Medical University (Dalian, China) between 2011 and 2013. The gastric tissues outside the cancer loci were selected as the tumor-adjacent tissue samples; 335 tumor-adjacent tissue samples from these patients were collected as controls. The diagnosis of gastric cancer was confirmed by pathological staining. Clinicopathological data including patient age, gender, tumor location, tumor size, histological differentiation, invasion depth, Helicobacter pylori (H. pylori) infection, ascites, lymphatic invasion, lymph node metastasis, distant metastasis and tumor-node-metastasis (TNM) stage (26) were retrospectively retrieved from the medical records. The patients did not undergo radiotherapy or chemotherapy prior to surgery. Outcomes of interest included the overall survival (OS) and disease-free survival (DFS) rates. OS was defined as the duration from diagnosis to the last follow-up, or to mortality. DFS was calculated as the time from the initial diagnosis to local recurrence, or distant metastasis.

The patients consented to have tissue samples collected at the time of admission for surgery, according to protocols authorized by the Regional Human Ethics Committee of Dalian Medical University. The Medical Ethics Committee of Dalian Medical University approved the present study. Due to the retrospective nature of the present study, the Ethics Committees waived the requirement for written informed consent from the patients.

Tissue microarray (TMA)-based immunohistochemistry. Tissue cores were extracted from formalin-fixed and paraffin-embedded tissue blocks containing the tumor tissue samples and the tumor-adjacent tissue samples and arrayed into a paraffin recipient block. Sections ( $4 \mu \mathrm{m}$ thick) were obtained from the TMA blocks using a microtome, mounted on poly-L-lysine-coated glass slides and used for immunohistochemistry.

TMA sections were washed in xylene, rehydrated in a graded ethanol series and washed in tap water. The tissue sections were then heated in $100 \mathrm{ml} 10 \mathrm{mM}$ sodium citrate buffer ( $\mathrm{pH}$ 6.0) in a microwave oven (high power, $700 \mathrm{~W}$ for $3 \mathrm{~min}$; medium power, $400 \mathrm{~W}$ for $3 \mathrm{~min}$; low power, $100 \mathrm{~W}$ for $3 \mathrm{~min}$; a total of $9 \mathrm{~min}$ ) to retrieve antigen. The sections were then incubated at $37^{\circ} \mathrm{C}$ for $30 \mathrm{~min}$ in $3 \% \mathrm{H}_{2} \mathrm{O}_{2} /$ methanol to block endogenous peroxidase activity. Nonspecific protein binding sites were blocked by $10 \%$ normal goat serum (Boster Biological Technology, Ltd., Wuhan, China) at $37^{\circ} \mathrm{C}$ for $30 \mathrm{~min}$. The sections were incubated in a primary polyclonal rabbit anti-human antibody against PARP-1 (cat. ab6079; dilution 1:200; Abcam, Cambridge, UK) overnight at $4^{\circ} \mathrm{C}$, and subsequently incubated with a biotinylated goat anti-rabbit secondary antibody (cat. no. SAEP031; dilution, 1:150; Wuhan Elabscience Biotechnology Co., Ltd., Wuhan, China) for $30 \mathrm{~min}$ and streptavidin horseradish peroxidase (LSAB kit; Dako, Glostrup, Denmark) for an additional $30 \mathrm{~min}$. Sections were stained with 3,3-diaminobenzidine at room temperature for 50-60 sec, counterstained with hematoxylin, dehydrated with graded ethanol and mounted with neutral resin. For the negative controls, the primary antibodies were replaced with phosphate-buffered saline.

Evaluation of immunohistochemistry. Two pathologists blinded to the experimental conditions examined the final effective immunostaining under a light microscope (Eclipse 50i; Nikon Corporation, Tokyo, Japan). The intensity of immunoreactivity was graded on a scale of 0-3, as follows: 0) For no visible staining, i) for low staining, ii) for moderate staining and iii) for high staining. The percentage of the stained nuclei in gastric cancer gland cells and normal gland cells was assigned using 5\% increments. Five random, non-overlapping fields were defined and 40 cells for each field (a total of 200 cells) were selected in order to calculate the percentage of stained cells present in each sample. The immunoreactivity score (IRS) was determined by multiplying the intensity score and the percentage of stained nuclei, giving a minimum IRS score of 0 and a maximum of $300 \%$. Receiver operating characteristic (ROC) curve analysis was performed to determine an optimal cutoff IRS for PARP-1 expression. Plotting the sensitivity and specificity for each outcome under study generated the ROC curves.

Statistical analysis. The Pearson's $\chi^{2}$ test and the Fisher's exact probability test were used to determine significant differences between the categorical data. The Mann-Whitney U test was used to detect differences in the IRS or lymph node metastasis number between various groups. The Wilcoxon rank-sum test was used to compare the IRS of gastric cancer tissues with tumor-adjacent tissues, for the paired tissue samples. Kaplan-Meier survival plots were generated and comparisons between the survival curves were determined with a log-rank test. Cox's proportional hazards regression model was used to evaluate the association between the potential confounding variables and the prognosis (OS or DFS). Only those cases with complete data on all the variables $(n=153)$ were included in the multivariate analyses. The data were processed using SPSS 16.0 software (SPSS, Inc., Chicago, IL, USA). P<0.05 was considered to indicate statistical significance.

\section{Results}

Clinicopathological characteristics of patients with gastric cancer. The clinicopathological characteristics of 564 patients with gastric cancer are summarized in Table I. For these 564 patients, the features of tumor location of 498 patients (88.3\%), tumor size of 522 patients (92.6\%), histological differentiation of 555 patients (98.4\%), depth of tumor invasion of 529 patients $(93.8 \%)$, status of $H$. pylori infection of 297 patients $(52.7 \%)$, presence of ascites of 479 patients $(84.9 \%)$, lymphatic invasion of 540 patients $(95.7 \%)$, lymph 
node metastasis of 553 patients $(98.0 \%)$, distant metastasis of 434 patients $(77.0 \%)$ and TNM stage of 481 patients $(85.3 \%)$ were recorded. The average age (mean \pm standard deviation) of gastric cancer patients in the present study was $60.1 \pm 10.4$ years (range, 29-82 years). The histological differentiation of the cancers was determined in 555 patients as follows: $14.6 \%$ high differentiation $(n=81), 21.4 \%$ moderate differentiation, $(n=119)$ and $64.0 \%$ low differentiation $(n=355)$. The depth of tumor invasion was evaluated in 529 patients as follows: $10.8 \% \mathrm{~T} 1 \mathrm{n}=57$, tumor invades the mucosa or submucosa), $15.9 \%$ T2 ( $n=84$, tumor invades the muscularis propria), $64.1 \%$ T3 ( $n=339$, tumor invasion of the serosa), and 9.3\% T4 $(n=49$, tumor invades the adjacent organs and structures). The status of $H$. pylori infection was defined by a ${ }^{13} \mathrm{C}$-urea breath test. Of the total group, 208 patients $(70.0 \%)$ were diagnosed with $H$. pylori infection while 89 patients $(30.0 \%)$ were negative for $H$. pylori infection. The stage of the cancer was evaluated in 481 patients according to the TNM staging system as follows: $13.7 \%$ Stage I $(n=66), 24.9 \%$ stage II $(n=120), 35.3 \%$ stage III $(n=170)$ and $26.0 \%$ stage IV $(n=125)$. Ascites, lymphatic invasion, lymph node metastasis and distant metastasis occurred in $176(36.7 \%)$ of 479 patients, $203(37.6 \%)$ of 540 patients, $316(57.1 \%)$ of 553 patients and $148(34.1 \%)$ of 434 patients, respectively.

Follow-up information was available for 523 patients with gastric cancer. During the follow-up period of 0-95 months, relapses occurred in 246 cases and mortality occurred in 208 cases. The 5-year survival rate was determined to be $46.5 \%$. The mean OS time was 65.8 months (95\% confidence interval (CI); 62.6-68.9 months) and the mean DFS time was 60.5 months (95\% CI; 57.3-63.7 months) respectively.

PARP-1 overexpression in breast cancer. Using immunohistochemistry, the expression levels of PARP-1 in 564 gastric cancer tissue samples and 335 tumor-adjacent control tissue samples were studied. Positive PARP-1 staining in gastric tissues appeared as brown particles that were primarily nuclear (Fig. 1). The gastric cancer tissue samples exhibited significantly more intense staining for PARP-1 expression, as compared with tumor-adjacent tissue samples $(\mathrm{P}<0.001$; Fig. 2A). There was also a significant increase in the IRS of PARP-1 expression among paired samples ( $\mathrm{P}<0.001$; Fig. $2 \mathrm{~B})$.

The ROC analysis was performed to determine an optimal cutoff score for PARP-1 expression; the ROC curves for the clinicopathological features with statistical significance are indicated in Fig. 3. The area under the curve for the lymph node metastasis status had the largest relative area (Fig. 3A). Based on this outcome, a cutoff score of $175 \%$ was selected for PARP-1 expression levels: The low and high expression levels of PARP-1 were defined as IRS $\leq 175 \%$ and IRS $>175 \%$, respectively. A total of $298(52.8 \%)$ tumors exhibited low expression levels of PARP-1 and 266 (47.2\%) tumors exhibited high expression levels of PARP-1.

Association of PARP-1 expression with clinicopathological characteristics of gastric cancer. The association between PARP-1 expression levels and the clinicopathological characteristics of gastric cancer were investigated (Table II). Statistical analysis indicated that PARP-1 expression levels were increased with the following: Decreased differentiation
Table I. Clinicopathological characteristics of patients with gastric cancer.

\begin{tabular}{|c|c|c|c|}
\hline Features & Total & Number & $\%$ \\
\hline Age (years) & 564 & & \\
\hline$\leq 60$ & & 296 & 52.5 \\
\hline$>60$ & & 268 & 47.5 \\
\hline Gender & 564 & & \\
\hline Male & & 405 & 71.8 \\
\hline Female & & 159 & 28.2 \\
\hline Tumor location & 498 & & \\
\hline Lower & & 257 & 51.6 \\
\hline Middle & & 199 & 40.0 \\
\hline Upper & & 42 & 8.4 \\
\hline Tumor size (cm) & 522 & & \\
\hline$\leq 5.0$ & & 240 & 46.0 \\
\hline$>5.0$ & & 282 & 54.0 \\
\hline Histological differentiation & 555 & & \\
\hline High & & 81 & 14.6 \\
\hline Moderate & & 119 & 21.4 \\
\hline Low & & 355 & 64.0 \\
\hline Invasion depth & 529 & & \\
\hline $\mathrm{T} 1$ & & 57 & 10.8 \\
\hline $\mathrm{T} 2$ & & 84 & 15.9 \\
\hline $\mathrm{T} 3$ & & 339 & 64.1 \\
\hline $\mathrm{T} 4$ & & 49 & 9.3 \\
\hline H. pylori infection & 297 & & \\
\hline Negative & & 89 & 30.0 \\
\hline Positive & & 208 & 70.0 \\
\hline Ascites & 479 & & \\
\hline Negative & & 303 & 63.3 \\
\hline Positive & & 176 & 36.7 \\
\hline Lymphatic invasion & 540 & & \\
\hline Negative & & 337 & 62.4 \\
\hline Positive & & 203 & 37.6 \\
\hline Lymph node metastasis & 553 & & \\
\hline Negative & & 237 & 42.9 \\
\hline Positive & & 316 & 57.1 \\
\hline Distant metastasis & 434 & & \\
\hline Negative & & 286 & 65.9 \\
\hline Positive & & 148 & 34.1 \\
\hline TNM stage & 481 & & \\
\hline I & & 66 & 13.7 \\
\hline II & & 120 & 24.9 \\
\hline III & & 170 & 35.3 \\
\hline IV & & 125 & 26.0 \\
\hline
\end{tabular}

H. pylori, Helicobacter pylori; TNM, tumor-node-metastasis.

$(\mathrm{P}<0.001)$, increased depth of invasion $(\mathrm{P}=0.037)$, presence of lymphatic invasion $(\mathrm{P}<0.001)$, presence of lymph node metastasis $(\mathrm{P}<0.001)$ and advanced TNM stage $(\mathrm{P}=0.015)$. 
Table II. Association of the expression of PARP-1 with clinicopathological features of gastric cancer.

High expression

Features

$\mathrm{n}$

$\%$

P-value

Age at diagnosis (years)

$\leq 60$

130

0.105

$>60$

136

43.9

Gender

Male

194

72

50.7

Female

Lower

104

Middle

Tumor size $(\mathrm{cm})$

$\leq 5.0$

108

$>5.0$

146

45.0

Histologic differentiation

High

Moderate

Low

Invasion depth

53.5

T1

T2

T3

T4

H. pylori

Negative

Positive

Ascites

Negative

Positive

Lymphatic invasion

Negative

Positive

40.4

Lymph node metastasis

Negative

58.1

Positive

Distant metastasis

57.0

Negative

Positive

TNM stage

$$
\text { I II }
$$

40.9

52.2

${ }^{a} \mathrm{P}$-value obtained from pearson chi-square or Fisher's exact test; ${ }^{\mathrm{b}}$ statistically significant $(\mathrm{P}<0.05)$; H. pylori, Helicobacter pylori; TNM, tumor-node-metastasis; PARP-1, poly (adenosine diphosphate-ribose) polymerase 1 .

In addition, high PARP-1 expression levels were increased among patients with $H$. pylori infection $(\mathrm{P}=0.032)$. The expression levels of PARP-1 were not identified to be significantly associated with age, gender, tumor location, tumor size, distal metastasis or presence of ascites in the patients $(\mathrm{P}>0.05)$. 
Table III. Univariate Cox regression analysis of clinicopathological data associated with OS and DFS in gastric cancer.

\begin{tabular}{|c|c|c|c|c|c|}
\hline \multirow[b]{2}{*}{ Factors } & \multirow[b]{2}{*}{$\mathrm{n}$} & \multicolumn{2}{|l|}{ OS } & \multicolumn{2}{|l|}{ DFS } \\
\hline & & RR $(95 \%$ CI $)$ & P-value & RR $(95 \%$ CI $)$ & P-value \\
\hline Age, years & 523 & & $0.013^{\mathrm{a}}$ & & $0.005^{\mathrm{a}}$ \\
\hline$\leq 60 />60$ & & $1.412(1.075-1.856)$ & & $1.428(1.111-1.835)$ & \\
\hline Gender & 523 & & $0.017^{\mathrm{a}}$ & & $0.025^{\mathrm{a}}$ \\
\hline Female/male & & $0.673(0.486-0.932)$ & & $0.715(0.532-0.959)$ & \\
\hline Tumor location & 457 & & 0.732 & & 0.905 \\
\hline Upper/middle/lower & & $1.041(0.828-1.307)$ & & $1.013(0.822-1.247)$ & \\
\hline Tumor size, $\mathrm{cm}$ & 484 & & $<0.001^{\mathrm{a}}$ & & $<0.001^{\mathrm{a}}$ \\
\hline$>5.0 / \leq 5.0$ & & $1.958(1.460-2.624)$ & & $1.896(1.453-2.475)$ & \\
\hline Histologic differentiation & 514 & & $0.018^{\mathrm{a}}$ & & $0.011^{\mathrm{a}}$ \\
\hline High/moderate/low & & $0.779(0.633-0.959)$ & & $0.785(0.650-0.947)$ & \\
\hline Invasion depth & 495 & & $0.005^{\mathrm{a}}$ & & $0.003^{\mathrm{a}}$ \\
\hline $\mathrm{T} 4 / \mathrm{T} 3 / \mathrm{T} 2 / \mathrm{T} 1$ & & $1.317(1.088-1.596)$ & & $1.309(1.097-1.561)$ & \\
\hline H. pylori & 268 & & 0.172 & & $0.048^{\mathrm{a}}$ \\
\hline Positive/negative & & $1.341(0.880-2.045)$ & & $1.492(1.00-2.218)$ & \\
\hline Ascites & 439 & & $0.001^{\mathrm{a}}$ & & $<0.001^{\mathrm{a}}$ \\
\hline Yes/no & & $1.622(1.221-2.155)$ & & $1.623(1.249-2.107)$ & \\
\hline Lymphatic invasion & 500 & & 0.290 & & 0.102 \\
\hline Yes/no & & $1.164(0.879-1.541)$ & & $1.240(0.958-1.605)$ & \\
\hline Lymph node metastasis & 512 & & $<0.001^{\mathrm{a}}$ & & $<0.001^{\mathrm{a}}$ \\
\hline Yes/no & & $4.991(3.476-7.165)$ & & $4.979(3.594-6.898)$ & \\
\hline Distant metastasis & 400 & & 0.263 & & 0.116 \\
\hline Yes/no & & $1.192(0.876-1.623)$ & & $1.257(0.945-1.671)$ & \\
\hline TNM stage & 442 & & $<0.001^{\mathrm{a}}$ & & $<0.001^{\mathrm{a}}$ \\
\hline IV/III/II/I & & $1.524(1.309-1.775)$ & & $1.493(1.299-1.715)$ & \\
\hline PARP-1 expression & 523 & & $<0.001^{\mathrm{a}}$ & & $0.001^{\mathrm{a}}$ \\
\hline Positive/negative & & $1.685(1.280-2.218)$ & & $1.507(1.172-1.936)$ & \\
\hline
\end{tabular}

RR and 95\% CI were assessed using univariate Cox regression analysis; ${ }^{a}$ statistically significant $(\mathrm{P}<0.05)$; OS, overall survival; DFS, disease-free survival; RR, relative risk; CI, confidence interval; H. pylori, Helicobacter pylori; TNM, tumor-node-metastasis; PARP-1, poly (adenosine diphosphate-ribose) polymerase 1 .

The association between PARP-1 expression levels and lymph node metastasis in gastric cancer patients was also investigated (Fig. 4). The numbers of lymph nodes positive for metastasis were recorded from 553 gastric cancer patients, and ranged from 0 to 33 lymph nodes among the 553 patients. Compared with PARP-1 low expression levels, PARP-1 high expression levels were associated with a larger number of positive lymph nodes ( $\mathrm{P}<0.001 ;$ Fig. 4A). Furthermore, lymph node metastasis-positive tumors exhibited a higher IRS of PARP-1 expression $(\mathrm{P}<0.001$; Fig. 4B).

Association of PARP-1 expression with the survival of patients with gastric cancer. Kaplan-Meier analysis and a log-rank test were used to evaluate the association between the expression levels of PARP-1 protein and patient mortality. High expression levels of PARP-1 were associated with significantly shorter OS time $(\mathrm{P}<0.001$; Fig. 5A) and DFS time $(\mathrm{P}=0.001$; Fig. 5B).
Univariate analysis of the potential prognostic impact of the clinicopathological parameters identified age, gender, tumor size, histological differentiation, invasion depth, presence of ascites, lymph node metastasis, TNM stage and PARP-1 expression as significantly associated with OS and DFS in gastric cancer patients (Table III). Subsequently, multivariate Cox regression models (using the same clinicopathological features) revealed that only histological differentiation, presence of ascites, lymph node metastasis and TNM stage remained as independent prognostic factors (Table IV).

Subgroup analysis of the association between PARP-1 expression levels and the survival of gastric cancer patients. The present study used Kaplan-Meier analysis to investigate the association of PARP-1 expression levels with OS and DFS in subgroups of gastric cancer patients, which were categorized according to clinicopathological parameters. The results of the 
Table IV. Multivariate Cox regression analysis of OS and DFS in gastric cancer.

\begin{tabular}{|c|c|c|c|c|}
\hline \multirow[b]{2}{*}{ Factors } & \multicolumn{2}{|l|}{ OS } & \multicolumn{2}{|c|}{ DFS } \\
\hline & $\mathrm{RR}(95 \% \mathrm{CI})$ & $\mathrm{P}$ & $\mathrm{RR}(95 \% \mathrm{CI})$ & $\mathrm{P}$ \\
\hline Age, years $(\leq 60 />60)$ & $1.063(0.676-1.669)$ & 0.792 & $1.144(0.753-1.738)$ & 0.528 \\
\hline Gender (female/male) & $0.855(0.516-1.417)$ & 0.543 & $0.904(0.568-1.438)$ & 0.670 \\
\hline Tumor size, $\mathrm{cm}(>5.0 / \leq 5.0)$ & $1.447(0.838-2.499)$ & 0.185 & $1.346(0.815-2.222)$ & 0.246 \\
\hline Histological differentiation & $1.435(1.012-2.033)$ & $0.042^{\mathrm{a}}$ & $1.376(1.001-1.891)$ & $0.049^{\mathrm{a}}$ \\
\hline Invasion depth (T4/T3/T2/T1) & $1.136(0.796-1.621)$ & 0.483 & $1.218(0.879-1.689)$ & 0.236 \\
\hline H.pylori (positive/negative) & $1.260(0.740-2.144)$ & 0.395 & $1.366(0.838-2.225)$ & 0.211 \\
\hline Ascites (yes/no) & $1.614(1.011-2.576)$ & $0.045^{\mathrm{a}}$ & $1.686(1.098-2.588$ & $0.017^{\mathrm{a}}$ \\
\hline Lymph node metastasis (yes/no) & $3.432(1.784-6.603)$ & $<0.001^{\mathrm{a}}$ & $4.010(2.204-7.295)$ & $<0.001^{\mathrm{a}}$ \\
\hline TNM stage (IV/III/II/I) & $1.411(1.057-1.883)$ & $0.019^{\mathrm{a}}$ & $1.402(1.080-1.821)$ & $0.011^{\mathrm{a}}$ \\
\hline PARP-1 expression & $1.642(0.994-2.712)$ & 0.053 & $1.354(0.863-2.122)$ & 0.187 \\
\hline
\end{tabular}

$\mathrm{n}=153$; RR and 95\% CI were assessed using multivariate Cox regression analysis; ' statistically significant $(\mathrm{P}<0.05)$; OS, overall survival; DFS, disease free survival; RR, relative risk; CI, confidence interval; H. pylori, Helicobacter pylori; TNM, tumor-node-metastasis; PARP-1, poly (adenosine diphosphate-ribose) polymerase 1.

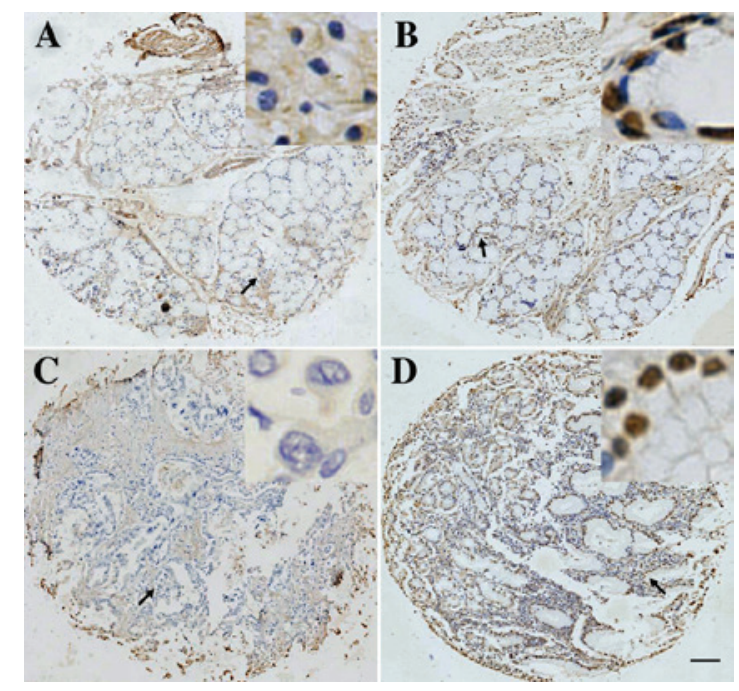

Figure 1. Representative micrographs indicating the immunohistochemical staining of PARP-1 proteins in gastric cancer tissue samples and in tumor-adjacent tissue samples. (A) Negative staining of PARP-1 in tumor-adjacent tissue samples and (B) gastric cancer tissue samples. (C) Positive staining of PARP-1 in tumor-adjacent tissue samples and (D) gastric cancer tissue samples. Magnification, x100. Scale bar, $100 \mu \mathrm{m}$. Arrows indicate the magnified regions in the insert (x1,000). PARP-1, poly (adenosine diphosphate-ribose) polymerase 1.

current study indicated that high expression levels of PARP-1 were associated with shorter OS $(\mathrm{P}<0.001$; Fig. 6A) and DFS $(\mathrm{P}<0.001$; Fig. 6B) in the subset of patients with $H$. pylori infection. However, in those patients without $H$. pylori infection, the expression levels of PARP-1 were not significantly associated with OS or DFS (OS, $\mathrm{P}=0338$; DFS, $\mathrm{P}=0.999$; Fig. 6C and D). In addition, high expression levels of PARP-1 were associated with shorter OS $(\mathrm{P}=0.001$; Fig. 7A) and DFS $(\mathrm{P}=0.006$; Fig. 7B) in the subset of patients with an advanced TNM stage, but not with an early TNM stage (OS, $\mathrm{P}=0.834$; DFS, P=0.955; Fig. 7C and D). The impact of PARP-1 expression levels on the prognosis was not significantly different between the subgroups of gastric cancer patients categorized according to age, gender, tumor size, tumor location, invasion depth, histological differentiation, presence of ascites, distant metastasis, lymphatic invasion or lymph node metastasis.

\section{Discussion}

PARP-1 is the most abundant and best characterized nuclear enzyme of the PARP superfamily (27). PARP-1 binds singleor double-stranded DNA breaks in response to stresses and functions to maintain genomic integrity (10). This role has been the focus of a number of studies in the field of oncology $(28,29)$. However, the role of PARP-1 in gastric cancer tumorigenesis remains to be determined. The present study investigated PARP-1 expression in gastric cancer for the first time. The present study has demonstrated that gastric cancer tissues exhibit significantly higher immunoreactivity of PARP-1, compared with tumor-adjacent tissues, indicating that PARP-1 overexpression may contribute to gastric cancer malignancy. These results are consistent with those of previous studies, which demonstrated that PARP-1 was upregulated in numerous types of tumor (11,15,30-32).

The present study analyzed the association between PARP-1 expression levels and various clinicopathological features in patients with gastric cancer. It was demonstrated that increased PARP-1 expression levels are associated with increased depth of invasion, lymphatic invasion, lymph node metastasis and advanced TNM stage. Furthermore, high PARP-1 expression levels were associated with a larger number of lymph node metastases, suggesting that PARP-1 over-expression increased invasion and metastasis in gastric cancer. Concordant with these results, Rodríguez et al (33) previously demonstrated that inhibition of PARP-1 expression suppressed the invasion and colonization of distal organs in melanoma cells. In addition, Li et al (34) demonstrated that inhibition of PARP expression attenuated the adhesion of mouse colon carcinoma cells to the extracellular matrix and decreased their 
A

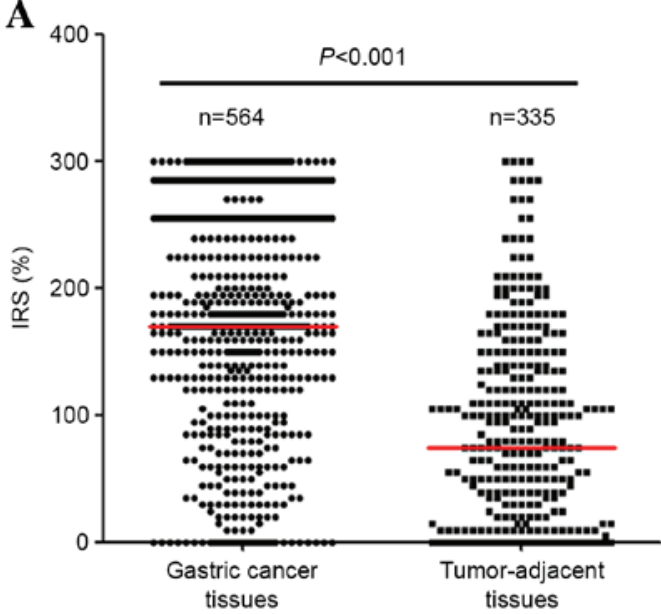

B

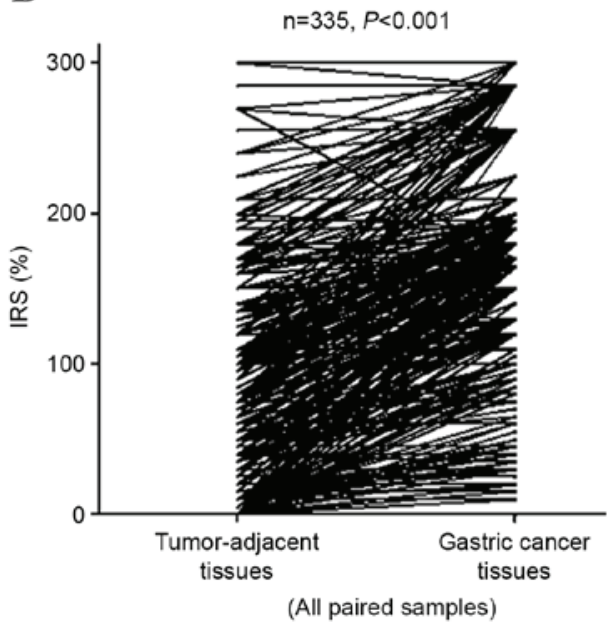

Figure 2. The difference in PARP-1 IRS between gastric cancer tissue samples and tumor-adjacent tissue samples. (A) The distribution of staining by IRS; the red line denotes the median value; the P-value was obtained using a Mann-Whitney U test. (B) Each line represents the IRS for an individual gastric cancer case with tumor-adjacent tissue samples and cancer tissue samples; P-values were obtained from a Wilcoxon rank-sum test. PARP-1, poly (adenosine diphosphate-ribose) polymerase 1 ; IRS, immunoreactivity score.
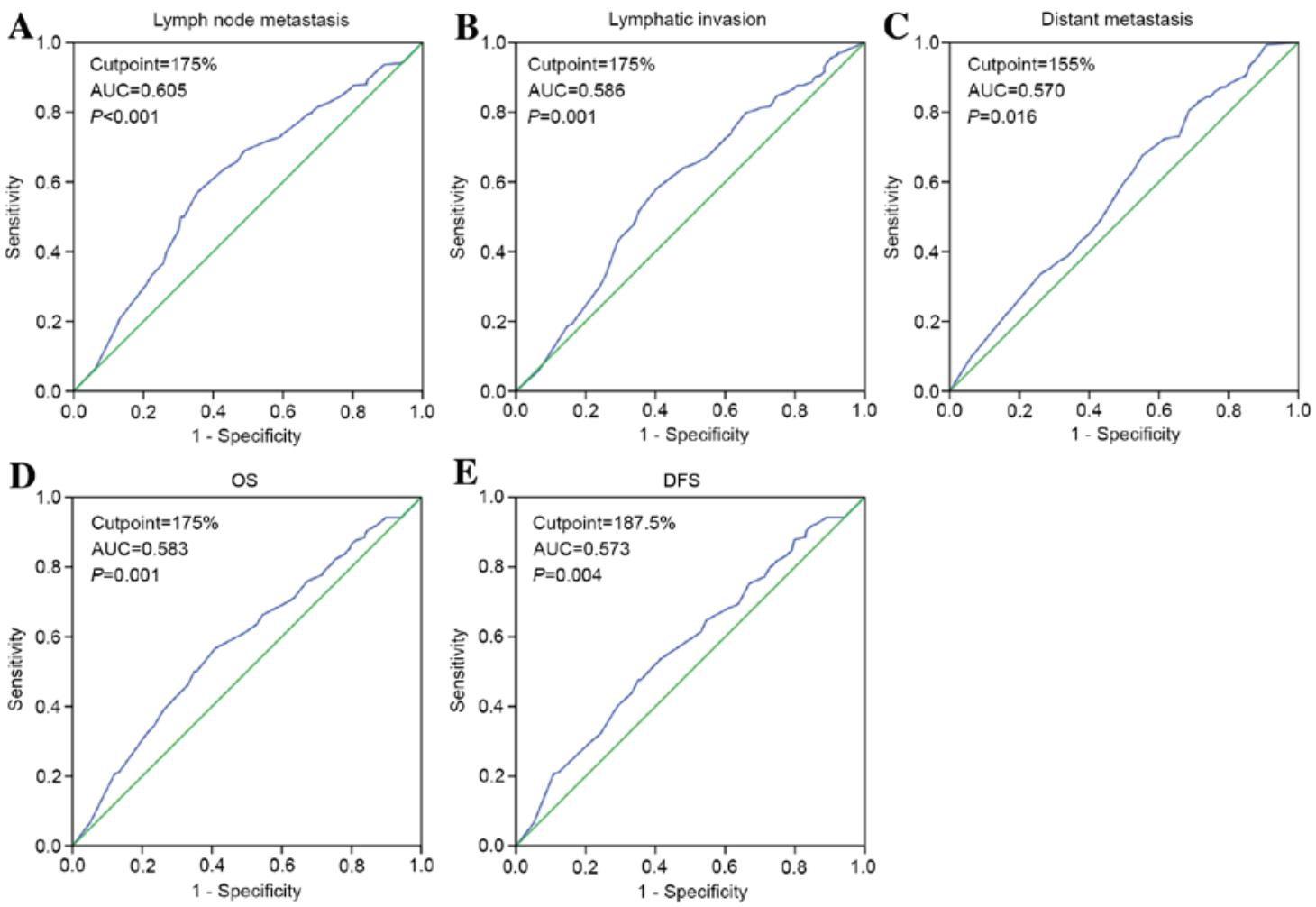

Figure 3. ROC curves were used to determine the cutoff for the IRS of PARP-1 expression based on the (A) lymph node metastasis status, (B) lymphatic invasion status, (C) distant metastasis status, (D) OS status and (E) DFS status of breast cancer patients. The sensitivity and specificity for each outcome were plotted and the AUCs and P-values were indicated. PARP-1, poly (adenosine diphosphate-ribose) polymerase 1; ROC, receiver operating characteristic; IRS, immunoreactivity score; AUCs, areas under curve.

migration and invasion through Matrigel, suggesting that PARP-1 is important in controlling the migration and invasion of certain cancers. PARP-1 has been previously demonstrated to regulate cell invasion and metastasis through the modulation of epithelial-mesenchymal transition-induced malignant transformation or the regulation of the activity of the nuclear factor kappa-light-chain-enhancer of activated B cells (33-36).
Further studies are required to determine whether a similar mechanism of PARP-1 occurs in gastric cancer.

The present study demonstrated that increased PARP-1 expression levels were associated with lower histological differentiation in gastric cancer. This result is concordant with the previously demonstrated inverse correlation between the degree of cell differentiation and PARP-1 activity (37). 
A

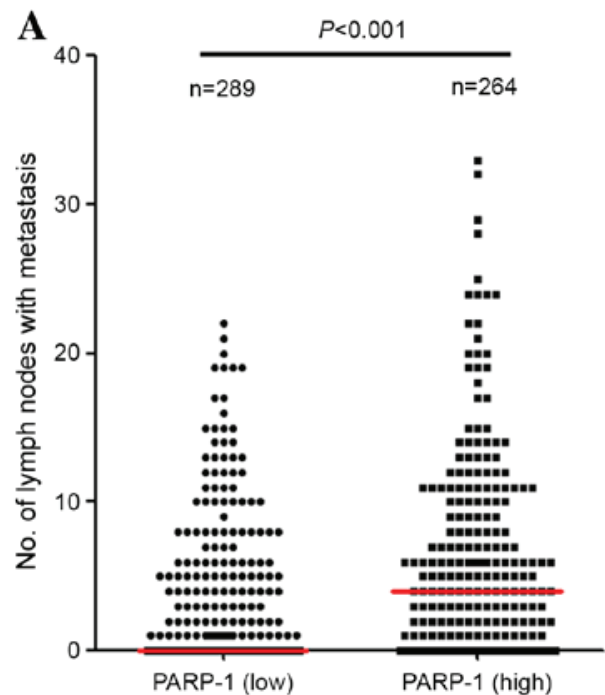

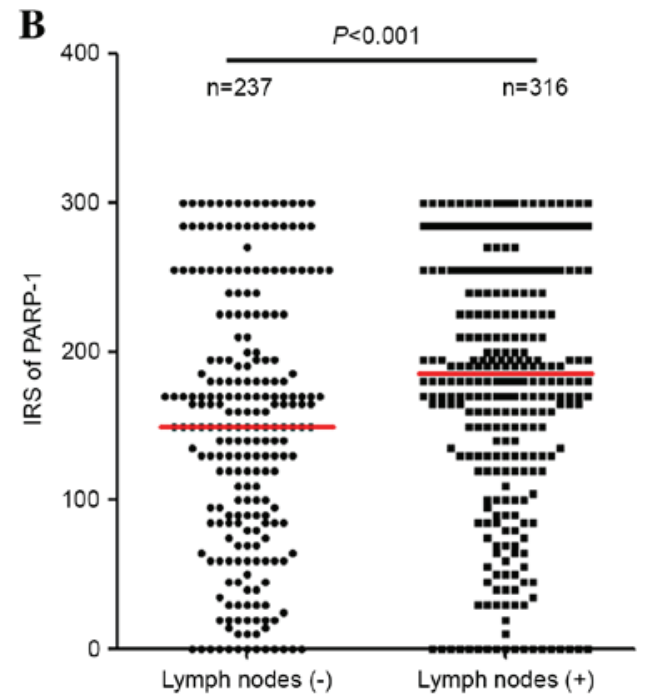

Figure 4. The association of PARP-1 expression with lymph node metastasis in patients with gastric cancer. (A) The number of metastasis-positive lymph nodes in cases of gastric cancer with high PARP-1 expression was increased significantly compared with those with low PARP-1 expression; (B) The IRS of cases of gastric cancer with positive lymph nodes was increased significantly compared with cases without positive lymph nodes. The red line denotes the median value. The P-values indicated in A and B were obtained using a Mann-Whitney U test. PARP-1, poly (adenosine diphosphate-ribose) polymerase 1; IRS, immunoreactivity score.

A

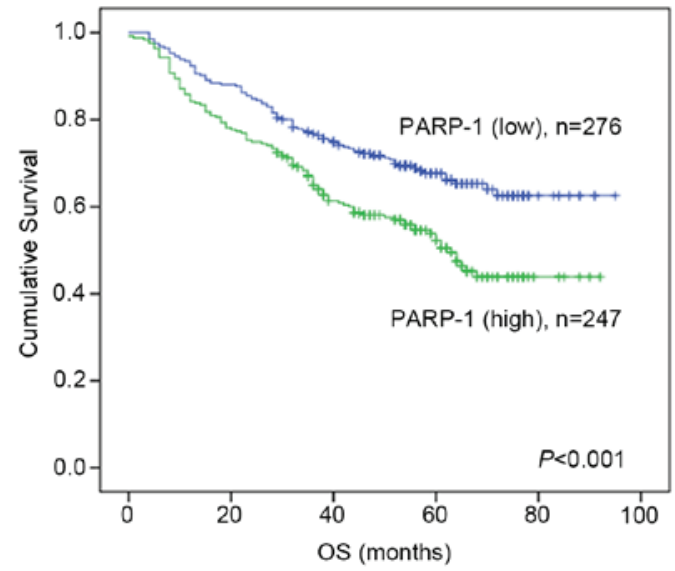

B

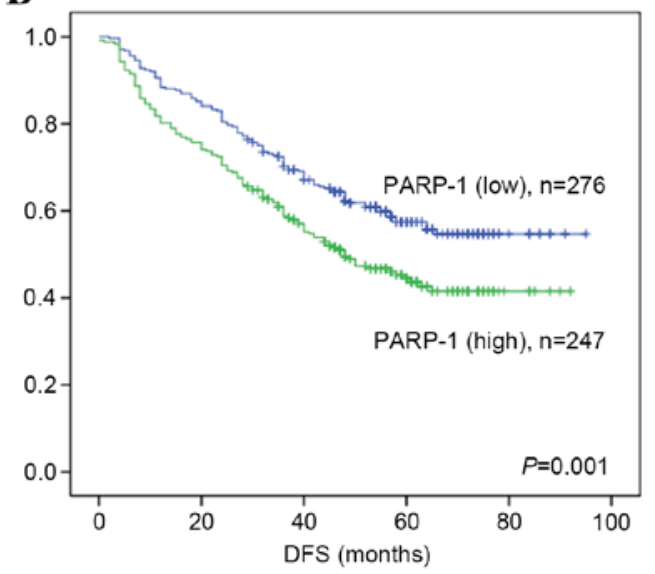

Figure 5. Kaplan-Meier estimates of gastric cancer patients stratified by the expression of PARP-1. Survival rate curves indicating that PARP-1 expression levels were significantly associated with a shorter (A) OS and (B) DFS. The log-rank test was performed to test the statistical significance. PARP-1, poly (adenosine diphosphate-ribose) polymerase 1; OS, overall survival; DFS, disease-free survival.

Barboro et al (38) demonstrated that higher PARP expression levels were detected in a less-differentiated PC3 cell line, as compared with a more-differentiated $\mathrm{LNCaP}$ prostate carcinoma cell line. It is possible that PARP-1 upregulation is involved in maintaining the stemness of cells, therefore exhibiting an association with lower differentiation in certain cancer cells. Concordant with this hypothesis, Chiou et al (39) previously demonstrated that the activation of PARP-1 promoted induced pluripotent stem cell production and helped to maintain a pluripotent state.

PARP-1 expression has been previously demonstrated to be associated with the poor prognosis of numerous tumor types, including early breast cancer and non-small cell lung cancer (13,40-42). However, Aiad et al (43) demonstrated that high nuclear PARP-1 expression levels were significantly associated with improved OS in locally advanced breast cancer;
Klauschen et al (44) demonstrated that low nuclear expression levels of PARP were associated with a poor prognosis in pancreatic cancer. These previous studies indicated that PARP-1 expression had differing roles in between different tumor types and stages of the tumors. The present study demonstrated that high PARP-1 expression levels are associated with significantly reduced DFS and OS in gastric cancer patients. Furthermore, high expression levels of PARP-1 were demonstrated to be associated with a poor prognosis in a subset of patients with an advanced TNM stage (III-IV), but not early TNM stage (I-II). In addition, a univariate Cox regression analysis identified that high PARP-1 expression levels are associated with a poor prognosis for gastric cancer patients. Therefore, PARP-1 expression levels may have a prognostic value in gastric cancer, particularly for those patients with an advanced TNM stage. However, a multivariate analysis 
A
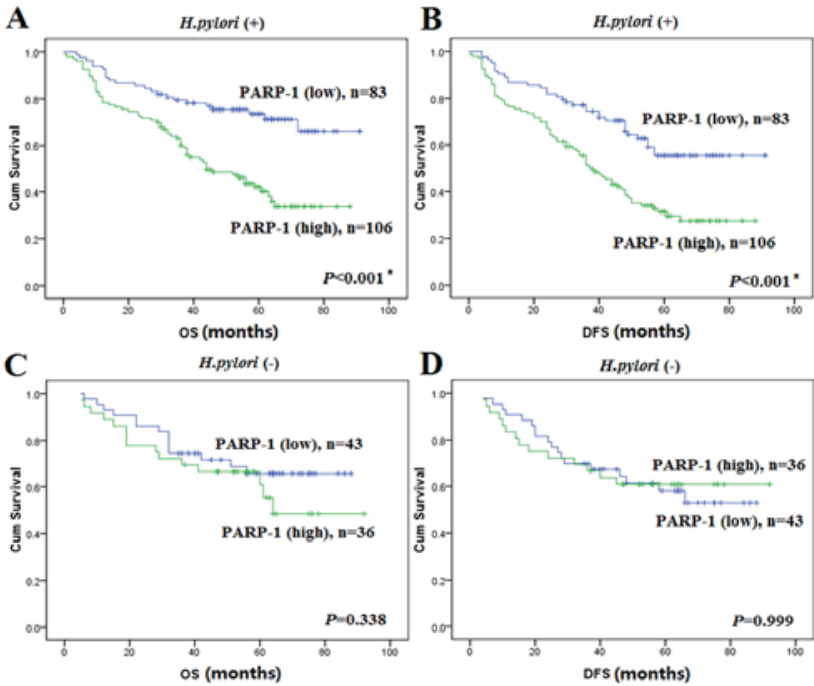

D

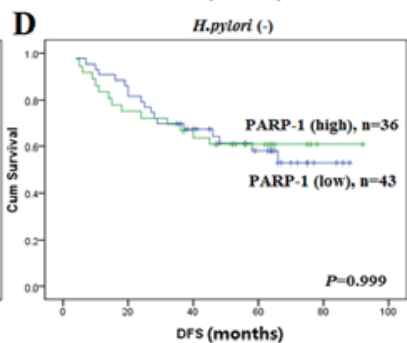

Figure 6. Analysis of the prognostic significance of PARP-1 expression levels in specific subgroups of breast cancer patients with or without H. pylori infection. Survival rate curves demonstrate that PARP-1 expression levels were significantly associated with (A) OS and (B) DFS in patients with $H$. pylori infection but were not significantly associated with shorter (C) OS or (D) DFS in patients without $H$. pylori infection. A log-rank test was performed to determine the statistical significance. "Indicated statistical significance. PARP-1, poly (adenosine diphosphate-ribose) polymerase 1 H. pylori, Helicobacter pylori; OS, overall survival; DFS, disease-free survival.

determined that PARP-1 expression levels were not independent prognostic factors in gastric cancer, which may be due to a significant association between PARP-1 overexpression and tumor invasion and metastasis of gastric cancer.

H. pylori may induce apoptosis of gastric epithelial cells $(45,46)$, and $H$. pylori infection is a risk factor associated with gastric cancer $(47,48)$. Chen et al $(49)$ previously demonstrated that incubating BGC-823 gastric cancer cells with $H$. pylori extract induced a breakdown of caspase- 1 and caspase-3, but not of PARP.Nossa et al (50) subsequently demonstrated that PARP-1 became activated in $H$. pylori infected gastric epithelial cells. Notably, the present study similarly demonstrated that PARP-1 expression levels were significantly increased in $H$. pylori infected gastric cancer cells. Regarding pathogenic infection, Hassumi-Fukasawa et al (51) demonstrated a significant positive association between PARP-1 expression levels and human papilloma virus positivity, in high-grade squamous intraepithelial lesions of the uterine cervix. Therefore, PARP-1 expression levels may be involved in host cell responses to pathogen infection. Furthermore, the current study demonstrated that the expression of PARP-1 is associated with significantly shorter OS and DFS in gastric cancer patients with $H$. pylori infection, but not in patients without $H$. pylori infection. These results suggest that the upregulation or activation of PARP-1 in response to $H$. pylori infection may be one mechanism underlying the association of PARP-1 expression levels and poor prognosis in gastric cancer patients. Concordant with a previous study (52), the results of the present study indicate the potential applications of PARP-1 targeted therapy for treating $H$. pylori-mediated gastric cancer.

In conclusion, the present study was a novel investigation into PARP-1 expression patterns in gastric cancer, and the
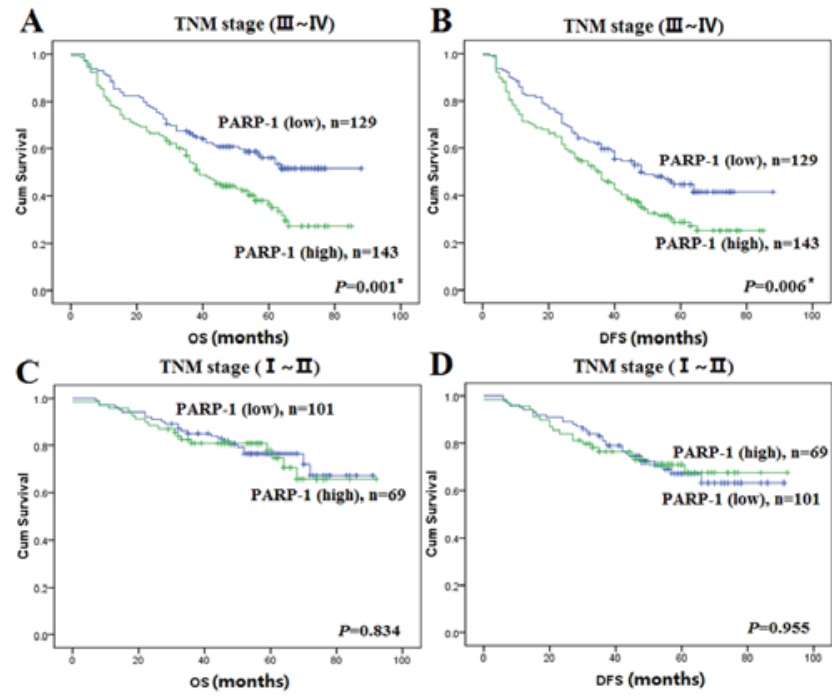

Figure 7. Analysis of the prognostic significance of PARP-1 in specific subgroups of breast cancer patients with various TNM stages. Survival rate curves show that high PARP-1 expression levels were significantly associated with shorter (A) OS or (B) DFS in patients with advanced TNM stage (III-IV) but not (C and D) early TNM stage (I-II). A log-rank test was performed to evaluate the statistical significance. Asterisks $\left(^{*}\right)$ indicated statistical significance. PARP-1, poly (adenosine diphosphate-ribose) polymerase 1; OS, overall survival; DFS, disease-free survival; TNM, tumor-node-metastasis

association between PARP-1 expression levels and the clinicopathological features and prognosis of gastric cancer patients. The results of the current study demonstrated that PARP-1 expression levels are significantly higher in gastric cancer tissues as compared with tumor-adjacent tissues; high PARP-1 expression levels are associated with $H$. pylori infection, lower histological differentiation, tumor invasion and metastasis, and poor prognosis in gastric cancer. Furthermore, it was demonstrated that high PARP-1 expression levels are associated with increased mortality in the subgroups of patients with H. pylori infection and an advanced TNM stage. The results of the present study suggest that the inhibition of PARP-1 may suppress tumor invasion and metastasis and improve histological differentiation and the survival rate in gastric cancer. The targeting PARP-1 may be an effective therapeutic strategy for the treatment of gastric cancer, particularly of H. pylori-positive or advanced-stage gastric cancer.

\section{Acknowledgements}

This study was supported by the Science and Technology Department Foundation of Liaoning Province (grant no. 2013225021).

\section{References}

1. Torre LA, Bray F, Siegel RL, Ferlay J, Lortet-Tieulent J and Jemal A: Global cancer statistics, 2012. CA Cancer J Clin 65: 87-108, 2015.

2. Lordick F, Allum W, Carneiro F, Mitry E, Tabernero J, Tan P, Van Cutsem E, van de Velde C and Cervantes A: Unmet needs and challenges in gastric cancer: The way forward. Cancer Treat Rev 40: 692-700, 2014.

3. Hakmé A, Wong HK, Dantzer F and Schreiber V: The expanding field of poly (ADP-ribosyl)ation reactions. 'Protein modifications: Beyond the usual suspects' review series. EMBO Rep 9: 1094-1100, 2008. 
4. Hottiger MO, Hassa PO, Luscher B, Schuler $H$ and Koch-Nolte F: Toward a unified nomenclature for mammalian ADP-ribosyltransferases. Trends Biochem Sci 35: 208-219, 2010.

5. Michels J, Vitale I, Saparbaev M, Castedo M and Kroemer G: Predictive biomarkers for cancer therapy with PARP inhibitors. Oncogene 33: 3894-3907, 2014.

6. Chambon P, Weill JD and Mandel P: Nicotinamide mononucleotide activation of new DNA-dependent polyadenylic acid synthesizing nuclear enzyme. Biochem Biophys Res Commun 11: 39-43, 1963.

7. Sugimura T and Miwa M: Poly (ADP-ribose): Historical perspective. Mol Cell Biochem 138: 5-12, 1994.

8. Davies JR, Jewell R, Affleck P, Anic GM, Randerson-Moor J, Ozola A, Egan KM, Elliott F, García-Casado Z, Hansson J, et al Inherited variation in the PARP1 geneand survival from melanoma. Int J Cancer 135: 1625-1633, 2014.

9. Bacalini MG, Di Lonardo D, Catizone A, Ciccarone F, Bruno T, Zampieri M, Guastafierro T, Calabrese R, Fanciulli M, Passananti C, et al: Poly (ADP-ribosyl)ation affects stabilization of Che-1 protein in response to DNA damage. DNA Repair (Amst) 10: 380-389, 2011.

10. Schreiber V, Dantzer F, Ame JC and de Murcia G: Poly (ADP-ribose): Novel functions for an old molecule. Nat Rev Mol Cell Biol 7: 517-528, 2006

11. Staibano S, Pepe S, Lo Muzio L, Somma P, Mascolo M, Argenziano G, Scalvenzi M, Salvatore G, Fabbrocini G, Molea G, et al: Poly(adenosine diphosphate-ribose) polymerase 1 expression in malignant melanomas from photoexposed areas of the head and neck region. Hum Pathol 36: 724-731, 2005

12. Nosho K, Yamamoto H, Mikami M, Taniguchi H, Takahashi T, Adachi Y, Imamura A, Imai K and Shinomura Y: Overexpression of poly(ADP-ribose) polymerase-1 (PARP-1) in the early stage of colorectal carcinogenesis. Eur J Cancer 42: 2374-2381, 2006.

13. Rojo F, Garcia-Parra J, Zazo S, Tusquets I, Ferrer-Lozano J, Menendez S, Eroles P, Chamizo C, Servitja S, Ramírez-Merino N, et al: Nuclear PARP-1 protein overexpression is associated with poor overall survival in early breast cancer. Ann Oncol 23: 1156-1164, 2012.

14. Mego M, Cierna Z, Svetlovska D, Macak D, Machalekova K Miskovska V, Chovanec M, Usakova V, Obertova J, Babal P and Mardiak J: PARP expression in germ cell tumours. J Clin Pathol 66: 607-612, 2013.

15. Sun Y, Gallacchi D, Zhang EY, Reynolds SB, Robinson L, Malinowska IA, Chiou TT, Pereira AM, Li C, Kwiatkowski DJ, et al: Rapamycin-resistant poly (ADP-ribose) polymerase-1 overexpression is a potential therapeutic target in lymphangioleiomyomatosis. Am J Respir Cell Mol Biol 51: 738-749, 2014

16. Weaver AN and Yang ES: Beyond DNA Repair: Additional functions of PARP-1 in cancer. Front Oncol 3: 290, 2013.

17. Rouleau M, Patel A, Hendzel MJ, Kaufmann SH and Poirier GG: PARP inhibition: PARP1 and beyond. Nat Rev Cancer 10: 293-301, 2010

18. Benafif $\mathrm{S}$ and Hall M: An update on PARP inhibitors for the treatment of cancer. Onco Targets Ther 8: 519-528, 2015.

19. O'Shaughnessy J, Osborne C, Pippen JE, Yoffe M, Patt D, Rocha C, Koo IC, Sherman BM and Bradley C: Iniparib plus chemotherapy in metastatic triple-negative breast cancer. $\mathrm{N}$ Engl J Med 364: 205-214, 2011

20. Kummar S, Ji J, Morgan R, Lenz HJ, Puhalla SL, Belani CP, Gandara DR, Allen D, Kiesel B, Beumer JH, et al: A phase I study of veliparib in combination with metronomic cyclophosphamide in adults with refractory solid tumors and lymphomas. Clin Cancer Res 18: 1726-1734, 2012.

21. Zhang Q, Li Y, Li X, Zhou W, Shi B, Chen H and Yuan W: PARP-1 Val762Ala polymorphism, $\mathrm{CagA}^{+} H$. pylori infection and risk for gastric cancer in Han Chinese population. Mol Biol Rep 36: 1461-1467, 2009.

22. Kim J, Pyun JA, Cho SW, Lee K and Kwack K: Lymph node metastasis of gastric cancer is associated with the interaction between poly (ADP-ribose) polymerase 1 and matrix metallopeptidase 2. DNA Cell Biol 30: 1011-1017, 2011.

23. He W, Liu T, Shan Y, Zhu K and Li Y: PARP1 polymorphisms increase the risk of gastric cancer in a Chinese population. Mol Diagn Ther 16: 35-42, 2012.

24. Le TV, Suh JH, Kim N and Park HJ: In silico identification of poly (ADP-ribose)polymerase-1 inhibitors and their chemosensitizing effects against cisplatin-resistant human gastric cancer cells. Bioorg Med Chem Lett 23: 2642-2646, 2013.
25. Liu HR, Meng LY, Lin ZY, Shen Y, Yu YQ and Zhu YZ: Cochinchina momordica seed extract induces apoptosis and cell cycle arrest in human gastric cancer cells via PARP and p53 signal pathways. Nutr Cancer 64: 1070-1077, 2012.

26. Fielding JW, Roginski C, Ellis DJ, Jones BG, Powell J, Waterhouse JA and Brookes VS: Clinicopathological staging of gastric cancer. Br J Surg 71: 677-680, 1984.

27. Krishnakumar R and Kraus WL: The PARP side of the nucleus: Molecular actions, physiological outcomes, and clinical targets. Mol Cell 39: 8-24, 2010.

28. Cepeda V, Fuertes MA, Castilla J, Alonso C, Quevedo C, Soto M and Pérez JM: Poly (ADP-ribose) polymerase-1 (PARP-1) inhibitors in cancer chemotherapy. Recent Pat Anticancer Drug Discov 1: 39-53, 2006.

29. Mégnin-Chanet F, Bollet MA and Hall J: Targeting poly(ADP-ribose) polymerase activity for cancer therapy. Cell Mol Life Sci 67: 3649-3662, 2010.

30. Shimizu S, Nomura F, Tomonaga T, Sunaga M, Noda M, Ebara M and Saisho H: Expression of poly(ADP-ribose) polymerase in human hepatocellular carcinoma and analysis of biopsy specimens obtained under sonographic guidance. Oncol Rep 12: 821-825, 2004.

31. Miwa M and Masutani M: PolyADP-ribosylation and cancer. Cancer Sci 98: 1528-1535, 2007.

32. Brustmann H: Poly(adenosine diphosphate-ribose) polymerase expression in serous ovarian carcinoma: Correlation with $\mathrm{p} 53$, MIB-1, and outcome. Int J Gynecol Pathol 26: 147-153, 2007.

33. Rodriguez MI, Peralta-Leal A, O'Valle F, Rodriguez-Vargas JM, Gonzalez-Flores A, Majuelos-Melguizo J, López L, Serrano S, de Herreros AG, Rodríguez-Manzaneque JC, et al: PARP-1 regulates metastatic melanoma through modulation of vimentin-induced malignant transformation. PLoS Genet 9: e1003531, 2013.

34. Li M, Threadgill MD, Wang Y, Cai L and Lin X: Poly(ADP-ribose) polymerase inhibition down-regulates expression of metastasis-related genes in CT26 colon carcinoma cells. Pathobiology 76: 108-116, 2009

35. Pu H, Horbinski C, Hensley PJ, Matuszak EA, Atkinson T and Kyprianou N: PARP-1 regulates epithelial-mesenchymal transition (EMT) in prostate tumorigenesis. Carcinogenesis 35: 2592-2601, 2014.

36. Lönn P, van der Heide LP, Dahl M, Hellman U, Heldin CH and Moustakas A: PARP-1 attenuates Smad-mediated transcription. Mol Cell 40: 521-532, 2010

37. Virág L and Szabó C: The therapeutic potential of poly(ADP-ribose) polymerase inhibitors. Pharmacol Rev 54 375-429, 2002.

38. Barboro P, Repaci E, D'Arrigo C and Balbi C: The role of nuclear matrix proteins binding to matrix attachment regions (Mars) in prostate cancer cell differentiation. PLoS One 7: e40617, 2012.

39. Chiou SH, Jiang BH, Yu YL, Chou SJ, Tsai PH, Chang WC, Chen LK, Chen LH, Chien Y and Chiou GY: Poly (ADP-ribose) polymerase 1 regulates nuclear reprogramming and promotes iPSC generation without c-Myc. J Exp Med 210: 85-98, 2013.

40. Xie KJ, He HE, Sun AJ, Liu XB, Sun LP and Dong XJ: Expression of ERCC1, MSH2 and PARP1 in non-small cell lung cancer and prognostic value in patients treated with platinum-based chemotherapy. Asian Pac J Cancer Prev 15: 2591-2596, 2014.

41. Donizy P, Pietrzyk G, Halon A, Kozyra C, Gansukh T, Lage H, Surowiak P and Matkowski R: Nuclear-cytoplasmic PARP-1 expression as an unfavorable prognostic marker in lymph node-negative early breast cancer: 15-year follow-up. Oncol Rep 31: 1777-1787, 2014.

42. Goncalves A, Finetti P, Sabatier R, Gilabert M, Adelaide J, Borg JP, Chaffanet M, Viens P, Birnbaum D and Bertucci F: Poly (ADP-ribose) polymerase-1 mRNA expression in human breast cancer: A meta-analysis. Breast Cancer Res Treat 127: 273-281, 2011.

43. Aiad HA, Kandil MA, El-Tahmody MA, Abulkheir IL, Abulkasem FM, Elmansori AA and Aleskandarany MA: The prognostic and predictive significance of PARP-1 in locally advanced breast cancer of Egyptian patients receiving neoadjuvant chemotherapy. Appl Immunohistochem Mol Morphol 23: 571-579, 2015.

44. Klauschen F, von Winterfeld M, Stenzinger A, Sinn BV, Budczies J, Kamphues C, Bahra M, Wittschieber D, Weichert W, Striefler J, et al: High nuclear poly-(ADP-ribose)-polymerase expression is prognostic of improved survival in pancreatic cancer. Histopathology 61: 409-416, 2012.

45. Moss SF, Calam J, Agarwal B, Wang S and Holt PR: Induction of gastric epithelial apoptosis by Helicobacter pylori. Gut 38: 498-501, 1996. 
46. Wagner S, Beil W, Westermann J, Logan RP, Bock CT, Trautwein C, Bleck JS and Manns MP: Regulation of gastric epithelial cell growth by Helicobacter pylori: Offdence for a major role of apoptosis. Gastroenterology 113: 1836-1847, 1997.

47. Atherton JC: The pathogenesis of Helicobacter pylori-induced gastro-duodenal diseases. Annu Rev Pathol 1: 63-96, 2006.

48. Polk DB and Peek RM Jr: Helicobacter pylori: Gastric cancer and beyond. Nat Rev Cancer 10: 403-414, 2010.

49. Chen Y, Wang Y, Xu W and Zhang Z: Analysis on the mechanism of Helicobacter pylori-induced apoptosis in gastric cancer cell line BGC-823. Int J Mol Med 16: 741-745, 2005.
50. Nossa CW, Jain P, Tamilselvam B, Gupta VR, Chen LF, Schreiber V, Desnoyers S and Blanke SR: Activation of the abundant nuclear factor poly(ADP-ribose) polymerase-1 by Helicobacter pylori. Proc Natl Acad Sci USA 106: 19998-20003, 2009.

51. Hassumi-Fukasawa MK, Miranda-Camargo FA, Zanetti BR, Galano DF, Ribeiro-Silva A and Soares EG: Expression of BAG-1 and PARP-1 in precursor lesions and invasive cervical cancer associated with human papillomavirus (HPV). Pathol Oncol Res 18: 929-937, 2012.

52. Nossa CW and Blanke SR: Helicobacter pylori activation of PARP-1: Usurping a versatile regulator of host cellular health. Gut Microbes 1: 373-378, 2010. 